\title{
openheart Coronary artery stenosis risk and time course in Kawasaki disease patients: experience at a US tertiary pediatric centre
}

\author{
Alok Sunil Patel, Margaret Bruce, Whitney Harrington, Michael A Portman
}

To cite: Patel AS, Bruce M, Harrington W, et al. Coronary artery stenosis risk and time course in Kawasaki disease patients: experience at a US tertiary pediatric centre. Open Heart 2015;2:e00206. doi:10.1136/openhrt-2014000206

Received 8 October 2014 Revised 2 January 2015 Accepted 19 January 2015

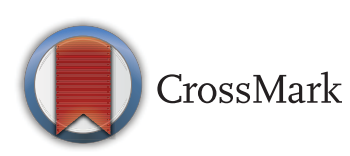

Department of Pediatrics, University of Washington, and Seattle Children's Research Institute, Seattle, Washington, USA

\section{Correspondence to} Dr Michael A Portman; Michael.Portman@ seattlechildrens.org

\section{ABSTRACT}

Objective: Despite treatment with intravenous immunoglobulin (IVIG), the natural progression to coronary artery stenosis in Kawasaki disease is not well defined and remains a potential cause of long-term morbidity. We present a novel study, at a US tertiary paediatric care centre, identifying risk factors for stenosis.

Methods: We reviewed charts of all children who underwent cardiac catheterisation for coronary artery abnormalities, from 1998 to January 2014, at a tertiary paediatric care centre. Demographic and diagnostic data included time intervals to echocardiographic changes and confirmed catheterisation cases of stenosis. Multivariate survival analysis was used to evaluate risk factors with stenosis formation as the main outcome measure.

Results: 53 children met the inclusion criteria and $18(34.6 \%)$ developed stenosis. Only those with giant coronary aneurysms (GCA) developed stenosis, with the highest risk group overall being children under the age of 6 months (hazard ratio (HR) 2.82 3.79, $\mathrm{p}=0.004)$. In a subset of only cases of GCA (33), a majority went on to develop stenosis (18/33). Median time to diagnosis was 190 days. In this group, children under the age of 6 months were again at highest risk (HR 2.62, $p=0.04)$. IVIG administration, sex and ethnicity were not statistically significant predictors.

Conclusions: This retrospective study demonstrates a relatively high incidence of stenosis in children with Kawasaki disease and coronary vascular abnormalities. Overall, a majority of cases with GCA progressed into stenosis, with children under the age of 6 months being at highest risk.

\section{INTRODUCTION}

Kawasaki disease (KD) is a self-limited vasculitis affecting medium-sized vessels, predominantly in children under the age of 5 years. Long-term complications include coronary aneurysms (CA) and secondary thrombotic and/or stenotic lesions. These lesions increase the risk of ischaemic heart disease and are important predictive factors for morbidity. ${ }^{1-3}$

\section{KEY MESSAGES}

What is already known about this subject?

- Previous retrospective studies identified large aneurysms in Kawasaki disease as a risk factor for long-term vascular complications, such as thrombotic events and stenosis. Additionally, young age is implicated as an independent risk factor for the development of aneurysms.

What does this study add?

- This study adds information about risk factors specific for the development of stenosis. We demonstrate that young age is an independent risk factor and furthermore, this study validates the association of giant coronary aneurysms and stenosis.

How might this impact on clinical practice?

- Given the risk of stenosis and aneurysms, we hope that Kawasaki disease will be included in the differential for any young child presenting with fever of unknown origin. Furthermore, all young children and/or children with giant coronary aneurysms warrant close follow-up and screening.

Without treatment, an estimated $15-25 \%$ of children with KD will develop aneurysms with a subsequent increased risk for potential myocardial infarction or ischaemic heart disease. $^{4} \quad{ }^{5}$ Treatment with intravenous immunoglobulin (IVIG) within the first 10 days of disease presentation decreases the incidence of aneurysms to $3-5 \% .^{6} \quad 7$ Additional risk factors for the development of CA include low presenting serum albumin, fever duration during acute phase, age less than 1 year or greater than 9 years, resistance to initial IVIG and delayed treatment. ${ }^{8-10}$

Transient CA or ectasia is a common finding in the first 10-14 days of the disease and usually spontaneously resolves. ${ }^{4}{ }^{11}$ The majority of patients with KD show the same cardiovascular risk as the general population. However, for those patients with significant 
and persistent coronary artery disease these risks still require definition. In particular, patients with persistent giant coronary artery aneurysms are classified within a high risk category by American Heart Association (AHA) guidelines. ${ }^{2}$ Shear stress at the entrance of chronic aneurysms triggers smooth muscle cells to transition into myofibroblasts, leading to laminar inflammation. ${ }^{12}{ }^{13}$ Larger aneurysms $(6 \mathrm{~mm})$ induce more remodelling and increase the risk of stenosis by $50 \% .{ }^{14} 15$

Young age is implicated in the development of giant coronary aneurysms (GCA), but little data exists about the natural progression from CA to stenosis. ${ }^{9}{ }^{16}$ In a Japanese population treated prior to the IVIG era, Kato et al found a $2.3 \%$ incidence of stenosis in the first year after disease and a subsequent increase to $4.7 \%$ over a 18-year surveillance time period. However, 12 of 26 patients from this cohort who had giant coronary artery aneurysms went on to develop stenoses or complete obstruction. Myocardial infarction occurred in 8 of these 12 patients, and 4 of these 8 died. Comparative data from a US centre with an ethnically heterogenous population and treated within the IVIG era has not been published. We present a study in an American population using multivariate survival models to identify risk factors for the development of stenosis as well as to determine the natural history and progression of the aneurysms.

\section{METHODS}

\section{Participants}

This is a retrospective cohort study of children with a diagnosis of $\mathrm{KD}$, findings of coronary artery dilation and surveillance cardiac catheterisation. This study was approved by the Seattle Children's Institutional Review Board. A total of 789 cases of Kawasaki disease, from 1998 to 2014, were reviewed from Seattle Children's Hospital. One hundred and thirty-one cases met Japanese Ministry of Health (JMH) criteria or had coronary artery z-scores suggestive of arterial abnormalities. ${ }^{17}$ Fifty-three of these individuals then met AHA guidelines described by Newburger $e t a l^{2}$ for cardiac catheterisation. These guidelines were published in 2004, but cases presenting before this date used a similar screening criteria, such as diffuse coronary ectasia or persistent aneurysms 6 months after initial diagnosis, to warrant catheterisation.

Initial KD diagnosis was based on published AHA criteria of fever $\geq 5$ days and $\geq 4$ classic clinical findings: dermatitis, conjunctival injection, extremity swelling, perioral erythema and/or lymphadenitis. ${ }^{2}$ Patients with incomplete criteria and coronary artery abnormalities were also included. Sex and age at diagnosis were noted for each patient. Treatment details noted included fever duration prior to initial IVIG, number of IVIG infusions and anticoagulation regimen, including drug trials. Time intervals between diagnosis, IVIG treatment and echocardiographic evidence of aneurysms were recorded. Time between onset of aneurysms and stenosis were also documented.
All patients received routine two-dimensional echocardiograms for coronary artery abnormality screening. As per the JMH criteria, any identified coronary artery diameters of $\geq 3 \mathrm{~mm}$ in children $<5$ years and $\geq 4 \mathrm{~mm}$ in children $\geq 5$ years were classified as abnormal. ${ }^{17}$ Aneurysms $\geq 6$ in children $\leq 1$ year of age and $\geq 8 \mathrm{~mm}$ at maximal diameter in children $>1$ year of age were classified as GCA.

Children meeting these criteria received coronary catheterisations, including cineangiography, to assess aneurysmal location, progression and to identify other potential anomalies of ascending aorta and left/right coronary systems. All angiographic observations during cardiac catheterisation were documented. Stenosis was noted when cineangiography showed complete occlusion or near occlusion ( $\sim 90 \%)$. In cases without a clear report of occlusion, multiple angiographic reports were reviewed to search for key terms related to stenosis formation, such as incomplete occlusion, evidence of collateral formation, poor dye perfusion and narrowing. Identified cases from key terms were then further investigated to make a judgment for inclusion.

\section{Statistical analysis}

Survival analysis was used to evaluate potential covariates predicting stenosis formation rates. End point was development of stenosis, with censored data secondary to different lengths of the clinical follow-up for each child.

Covariates in univariate and multivariate regression models include presence of GCA, age, sex and IVIG doses received. GCA and sex were modelled as dichotomous variables. Age was initially modelled as a categorical variable $(<6$ months, 6 months to 5 years, and $>5$ years) then was condensed into a dichotomous variable ( $<6$ months, $\geq 6$ months). IVIG (one dose, two or more doses and doses unknown) were categorical variables. Ethnicity was considered in initial analysis, but limited numbers within group sample size prohibited inclusion in final models.

Only children with GCA developed stenosis; therefore, multivariate analysis included only children with GCA. Age and IVIG doses were included in multivariate analysis as they were either independent predictors $(\alpha \leq 0.10)$ of stenosis or because they were potential confounders of the relationship between age and stenosis $(\geq 10 \%$ change in hazard ratio (HR)).

\section{RESULTS}

A total of 53 patients met the inclusion criteria for the study. The average age of KD diagnosis was $2.5 \pm 2.3$ years, with an age range 4 months to 11 years (table 1 ). Fifty patients received aspirin prophylaxis during the acute phase (three charts were incomplete). All children with GCA were anticoagulated with Coumadin.

From the entire sample, 18 individuals $(35.3 \%)$ developed stenosis over the entire observation phase of 5400 days. Median time of Kawasaki disease diagnosis to 


\begin{tabular}{|c|c|c|}
\hline Covariate & Number & Mean \\
\hline \multicolumn{3}{|l|}{ GCA } \\
\hline Present & 33 & 62.3 \\
\hline Absent & 20 & 37.74 \\
\hline \multicolumn{3}{|l|}{ Age } \\
\hline$\leq 6$ months & 8 & 15.1 \\
\hline$\overline{6}$ months to 5 years & 35 & 66 \\
\hline$>5$ years & 10 & 18.9 \\
\hline \multicolumn{3}{|l|}{ Sex } \\
\hline Male & 36 & 68 \\
\hline Female & 17 & 32.1 \\
\hline \multicolumn{3}{|l|}{ IVIG } \\
\hline 1 dose & 26 & 49.1 \\
\hline $2(+)$ doses & 22 & 42.3 \\
\hline Doses unknown & 5 & 9.6 \\
\hline
\end{tabular}

finding of stenosis was 190 days. Thirty-three individuals $(63.3 \%)$ had echocardiographic evidence of GCA (table 1). All cases of stenosis also had evidence of GCA; overall, 18 of $33(55 \%)$ of GCA cases developed stenosis. In subgroup survival analysis of individuals with GCA, a steady decline with stenosis as an end point can be observed over the entire observation phase (figure 1). The other 15 individuals with GCA were stenosis free on all follow-up catheterisations. $62.5 \%$ of stenosis cases occurred in children under 6 months, $33 \%$ in children aged 6 months to 5 years, and $20 \%$ in children greater than 5 years (table 1 ). One patient with giant coronary artery aneurysms suffered a myocardial infection, but there were no deaths.

Univarate analysis evaluated the relationship between potential predictors and risk of stenosis. Using children aged 6 months to 5 years as the reference group, children under the age of 6 months had a HR of 3.68

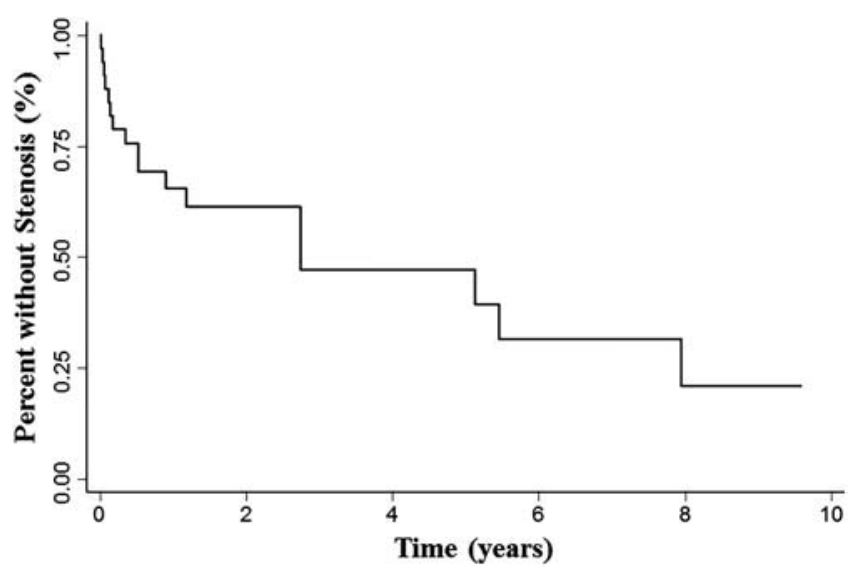

Figure 1 Survival analysis for individuals with giant coronary aneurysms (GCA). Kaplan-Meier for event free survival, with stenosis as end point among those individuals with GCA. $(\mathrm{p}=0.007)$, while children greater than 5 years of age had an HR of 0.88 ( $\mathrm{p}=0.87$; figure 2$)$. In post hoc analysis, those between 6 months and 5 years, and those greater than 5 years were not statistically different; therefore, the two were combined into a single group. With this combined group as a reference, children less than 6 months of age had a HR of $3.79(p=0.004)$. No HR could be calculated for presence of GCA, as only individuals with GCA developed stenosis. IVIG and gender were not significant predictors.

Participants without GCA were excluded from multivariate modelling as there were no cases of stenosis within this group. Among those with GCA, the final multivariate model considered age and IVIG to predict risk of stenosis. Only age $<6$ months was a significant risk factor for risk of stenosis (table 2). Cox-Regression analysis of individuals with GCA, with age as the covariate, showed a higher incidence of stenosis in the youngest age group of 6 months (figure 2). In this model, age group 6 months to 5 years was used as a reference.

\section{DISCUSSION}

Defining the risk of coronary artery stenosis in Kawasaki patients was the principal objective of this study. The analyses reveal a fairly high rate for development of stenoses in a US population with giant coronary artery aneurysms. The risk occurs despite treatment with IVIG and other secondary anti-inflammatory therapies. Furthermore, for the majority of these patients anticoagulation therapy conformed to current AHA guidelines. The data are consistent with results from a similar analysis performed in Quebec province showing 20/31 (65\%) patients with GCA developing either stenosis or complete obstruction of a coronary artery. ${ }^{18}$ Though our subject number was small, we also identified age $<6$ months in the setting of GCA as a significant risk factor.

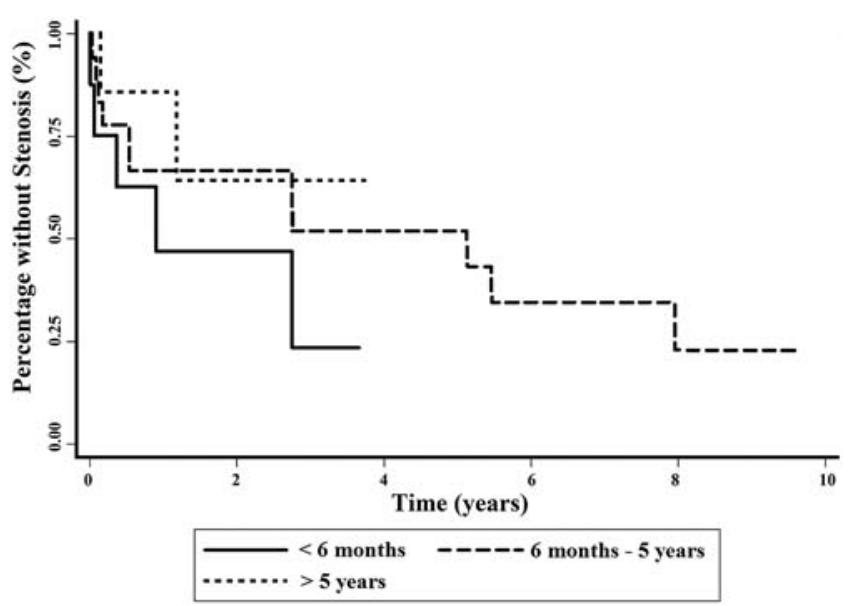

Figure 2 Failure curves of stenosis by age group.

Kaplan-Meier curve of age to predict event free survival with end point of stenosis. 
Table 2 Multivariate analysis to predict risk of stenosis among subset of patients with GCA

\begin{tabular}{llll}
\hline Covariate & HR & $95 \% \mathbf{C l}$ & p Value \\
\hline Age & & & \\
$>6$ months & Reference & $>6$ months & Reference \\
$\leq 6$ months & 2.62 & 1.04 to 6.63 & 0.04 \\
IVIG & & \\
1 dose & Reference & & \\
$2(+)$ doses & 1.28 & 0.38 to 4.27 & 0.69 \\
\hline $\begin{array}{l}\text { GCA, giant coronary aneurysms; IVIG, intravenous } \\
\text { immunoglobulin. }\end{array}$
\end{tabular}

The clinical import of these findings still requires clarification. A recent report, as well as the Quebec study, suggests that risk of cardiovascular events in the general Kawasaki population remains fairly low. ${ }^{17} 18$ A retrospective analysis compiled from a Kaiser Northern California population showed low aneurysm rates and few long-term adverse events in general KD population. ${ }^{19}$ We report near $7 \%$ prevalence of aneurysms for patients with $\mathrm{KD}$ seen at a major West Coast pediatric tertiary care centre, while the Kaiser study reported $<5 \%$. Thus, long- term follow-up for patients in our cohort may yield higher adverse event rates. Adverse event reporting alone, however, does not account for outcome modifying effects of therapeutic and preventative measures, which include close monitoring, aggressive anticoagulation and exercise restrictions for high-risk patients. We have identified exercise induced ischaemia in patients with KD with angiographically defined stenoses through either myocardial perfusion imaging or stress echocardiography. However, a clinical conundrum exists for those patients who are yet too young or too small to undergo revascularisation procedures.

Patients in our study with small and medium-size aneurysms did not develop coronary artery stenoses over the term of this study. This provides some reassurance for these patients and their parents. However, some intravascular imaging data suggest that coronary remodeling, due to intimal-medial wall thickness proliferation continues in the arteries, which were noted as $>4 \mathrm{~mm}$ during the acute phase of $\mathrm{KD} .{ }^{20}$ Isolated reports exist showing late obstruction in patients with regressed aneurysms. Thus, patients with dilation and regression require continued regular follow-up.

The retrospective nature of this study represents a limitation. At our centre, patients with coronary artery aneurysms generally undergo initial catheterisation with angiography within 12 months of presentation. However, some variability exists regarding repeat imaging studies and catheterisation. Furthermore, some patients may have been lost to follow-up or transferred to another location. Thus, we could have missed and underreported stenoses which developed over long time periods. We did define increase risk for the youngest subgroup. The small number of patients within this group may have also biased our results.
In conclusion, this retrospective study shows development of coronary artery stenoses in the majority of children with GCA over a long term. The patients $<6$ months of age remain at high risk and Cox-Proportional Hazard modelling suggests that these young children develop stenoses within 4 years. At less than 5 years of age, these stenoses are not generally considered amenable to revascularisation procedures. Thus, not only should these patients follow current regimens, but new therapies are required to arrest coronary artery remodelling after $\mathrm{KD}$.

Contributors ASP designed data collection methods, analysed data, devised statistical analysis, drafted and revised the paper. MAP designed the original data collection method, analysed data, drafted and revised the paper. He is guarantor of this paper. MB designed data collection methods, organised and oversaw all data collection. WH performed statistical analysis, contributed to tables and figures and revised the paper.

Competing interests None declared.

Ethics approval Seattle Children's Institutional Review Board.

Provenance and peer review Not commissioned; externally peer reviewed.

Open Access This is an Open Access article distributed in accordance with the Creative Commons Attribution Non Commercial (CC BY-NC 4.0) license, which permits others to distribute, remix, adapt, build upon this work noncommercially, and license their derivative works on different terms, provided the original work is properly cited and the use is non-commercial. See: http:// creativecommons.org/licenses/by-nc/4.0/

\section{REFERENCES}

1. Gersony WM. The adult after Kawasaki disease the risks for late coronary events. J Am Coll Cardiol 2009;54:1921-3.

2. Newburger JW, Takahashi M, Gerber MA, et al. Diagnosis, treatment, and long-term management of Kawasaki disease: a statement for health professionals from the Committee on Rheumatic Fever, Endocarditis and Kawasaki Disease, Council on Cardiovascular Disease in the Young, American Heart Association. Circulation 2004;110:2747-71.

3. Manlhiot C, Niedra E, McCrindle BW. Long-term management of Kawasaki disease: implications for the adult patient. Pediatr Neonatol 2013;54:12-21.

4. Kato $\mathrm{H}$, Sugimura $\mathrm{T}$, Akagi $\mathrm{T}$, et al. Long-term consequences of Kawasaki disease. A 10- to 21-year follow-up study of 594 patients. Circulation 1996;94:1379-85.

5. Dajani AS, Taubert KA, Gerber MA, et al. Diagnosis and therapy of Kawasaki disease in children. Circulation 1993;87:1776-80.

6. Newburger JW, Takahashi M, Burns JC, et al. The treatment of Kawasaki syndrome with intravenous gamma globulin. $N$ Engl J Med 1986;315:341-7.

7. Newburger JW, Takahashi M, Beiser AS, et al. A single intravenous infusion of gamma globulin as compared with four infusions in the treatment of acute Kawasaki syndrome. N Engl J Med 1991;324:1633-9.

8. Honkanen VEA, McCrindle BW, Laxer RM, et al. Clinical relevance of the risk factors for coronary artery inflammation in Kawasaki disease. Pediatr Cardiol 2003;24:122-6.

9. Manlhiot C, Yeung RSM, Clarizia NA, et al. Kawasaki disease at the extremes of the age spectrum. Pediatrics 2009;124:e410-15.

10. Sabharwal T, Manlhiot C, Benseler SM, et al. Comparison of factors associated with coronary artery dilation only versus coronary artery aneurysms in patients with Kawasaki disease. Am J Cardiol 2009;104:1743-7.

11. Manlhiot C, Millar K, Golding F, et al. Improved classification of coronary artery abnormalities based only on coronary artery z-scores after Kawasaki disease. Pediatr Cardiol 2010;31:242-9.

12. Orenstein JM, Shulman ST, Fox LM, et al. Three linked vasculopathic processes characterize Kawasaki disease: a light and transmission electron microscopic study. PLoS ONE 2012;7:e38998.

13. Suzuki A, Miyagawa-Tomita S, Komatsu K, et al. Active remodeling of the coronary arterial lesions in the late phase of Kawasaki disease: immunohistochemical study. Circulation 2000;101:2935-41. 
14. Tsuda E, Kamiya T, Ono $\mathrm{Y}$, et al. Incidence of stenotic lesions predicted by acute phase changes in coronary arterial diameter during Kawasaki disease. Pediatr Cardiol 2005;26:73-9.

15. Tsuda E, Kamiya T, Kimura K, et al. Coronary artery dilatation exceeding $4.0 \mathrm{~mm}$ during acute Kawasaki disease predicts a high probability of subsequent late intima-medial thickening. Pediatr Cardiol 2002;23:9-14.

16. Rosenfeld EA, Corydon KE, Shulman ST. Kawasaki disease in infants less than one year of age. J Pediatr 1995;126:524-9.

17. Research Committee on Kawasaki Disease. Report of subcommittee on standardization of diagnostic criteria and reporting of coronary artery lesions in Kawasaki disease. Tokyo, Japan: Japanese Ministry of Health and Welfare, 1984.

18. McNeal-Davidson A, Fournier A, Scuccimarri R, et al. The fate and observed management of giant coronary artery aneurysms secondary to Kawasaki disease in the Province of Quebec: the complete series since 1976. Pediatr Cardiol 2013;34:170-8.

19. Holve $T$, Patel $A$, Chau $Q$, et al. Long-term cardiovascular outcomes in survivors of Kawasaki disease. Pediatrics 2014;133:e305-311.

20. Lemura M, Ishii M, Sugimura $\mathrm{T}$, et al. Long term consequences of regressed coronary aneurysms after Kawasaki disease: vascular wall morphology and function. Heart 2000;83:307-11. 